\title{
Spontaneous rupture of the spleen in Legionnaires' disease
}

\author{
A.H. Holmes, V.W. Ng* and P. Fogarty** \\ The Intensive Care Unit, Whittington Hospital, Highgate Hill, London N19, UK.
}

\begin{abstract}
Summary: A case of Legionnaires' disease is described in a 63 year old man who presented with pneumonia and confusion. Eleven days after admission he became acutely hypotensive and attempts at resuscitation failed. Post-mortem examination revealed spontaneous splenic rupture and massive hepatocellular necrosis - an outcome that has not previously been associated with Legionnaires' disease.
\end{abstract}

\section{Introduction}

The multi-system involvement of Legionnaires' disease has been well documented. ${ }^{1-7}$ However, to our knowledge, spontaneous rupture of the spleen has not previously been described in association with this condition. We report a case in which this occurred.

\section{Case report}

A 63 year old male cleaner presented in February 1989 with a dry cough, dyspnoea, vomiting, confusion and rigors.

On examination he had a temperature of $39.5^{\circ} \mathrm{C}$, he was tachypnoeic and there were crackles at his left lung base. Chest X-ray showed extensive left-sided consolidation, and arterial blood gases showed hypoxia. Initial investigations demonstrated mild hyponatraemia; full blood count, urea, creatinine and liver function tests were normal. Legionnaires' disease was suspected, and was subsequently confirmed by detection of Legionella pneumophila antigen in his urine on day 3 and a four-fold rise in antibody titres. The organism was also isolated in his sputum.

Treatment was instituted with oxygen, and intravenous erythromycin and benzylpenicillin. The latter was stopped when the antigen was detected in his urine on day 3 . His condition deteriorated markedly over the next 12 hours; intravenous rifampicin was started, and he was intubated and ventilated. His renal function then declined and by

Correspondence: A.H. Holmes, M.A., M.R.C.P., Infectious Diseases Unit, The Churchill Hospital, Oxford, UK.

Present addresses: "The Brompton Hospital, Fulham Road, London SW3 6HP. "“c/o The Medical School, PO Box 885, Kingstown, St Vincent, West Indies.

Accepted: 9 May 1990 day 3 he required continuous haemofiltration. For 2 days he required inotrope support. By the seventh day he had been apyrexial for $\mathbf{4 8}$ hours. He was haemodynamically and biochemically stable and his chest was improving. However, he had become jaundiced and had developed tender hepatomegaly. The major biochemical abnormality was a rising level of bilirubin with mildly elevated transaminases. His prothrombin ratio was normal. Ultrasound showed a moderately enlarged liver and a normal biliary tree. The spleen was unremarkable. The rifampicin was stopped and he remained on erythromycin alone. On day 11 he became acutely hypotensive with no response to intravenous colloid and adrenaline. His abdomen became distended, but peritoneal aspiration revealed no fluid or blood. The patient died before any surgical intervention was possible.

Post-mortem examination demonstrated the cause of death to be rupture of the spleen. The spleen was enlarged, weighing 230 grams, and was soft and semi-fluid in substance. The splenic capsule had clearly been grossly distended and had ruptured. It was lying loose around the splenic pulp. A rupture had occurred along the anterior border extending into the hilar region of the spleen. Approximately $2 \frac{1}{2}$ litres of blood clot was found in the peritoneal cavity. His lungs demonstrated resolving consolidation, his liver was pale, swollen and congested, the adrenals were normal. Both kidneys were swollen and pale. Histological examination of the lungs revealed evidence of both resolving and organizing pneumonia with changes of adult respiratory distress syndrome. No Legionella could be identified on Dieterle's stain or on immunological testing of the normal fixed material. The spleen showed a mononuclear infiltrate of the pulp and a scanty polymorphonuclear infiltrate. Examination of the kidneys showed widespread tubular necrosis; the liver had suffered massive hepatocullar necrosis. 
Discussion

Spontaneous rupture of the spleen is known to be a rare complication of specific infectious diseases, notably typhoid fever, infectious mononucleosis and malaria, but has not been described in association with Legionnaires' disease.

Legionnaires' disease is a multi-system disease. Its involvement of the nervous system, musculoskeletal systems, gastrointestinal tract, kidneys, adrenals and the heart have been well documented. Spontaneous splenic rupture may be yet another complication of Legionella pneumophila infection. Although the cases of splenic rupture recorded in other infections are 'spontaneous', such rupture may be precipitated by minor or even trivial trauma. The patient described was exposed to frequent abdominal palpation and chest physiotherapy, which may conceivably have precipitated the splenic rupture in this case. Enlargement of the spleen is common in systemic infections. The enlarged, aroused spleen is then said to show a reactive hyperplasia. The splenomegaly is caused in part by the reactive hyperplasia and in part by congestion with erythrocytes. The enlarged spleen can become so engorged with erythrocytes that its natural rubbery resilience is lost and it becomes fragile and vulnerable to blunt trauma. This reactive hyperplasia maybe caused by the pathogenic organisms or the products of inflammation.

The morphology of the acute reactive hyperplasia is non-specific and does not enable an aetiological diagnosis to be made. However, in some infectious diseases there are modifications of the features, such as the presence of large numbers of atypical lymphocytes in infectious mononucleosis and the marked erythrophagia in typhoid fever. No specific splenic morphology has so far been described in Legionnaires' disease. Some of the splenic enlargement may be secondary to passive congestion due to intrahepatic derangement of portal venous drainage, and in this case the massive hepatic necrosis could have possibly contributed to this.

The massive hepatic necrosis was another unusual feature in this case, the cause of which is uncertain. It may have been due to the antibiotics, and has been described in association with rifampicin and less commonly erythromycin ${ }^{11}$ or it may have been due to legionella infection. Jaundice and elevation of liver enzymes have been reported in severe bacterial infection in the absence of local infection. ${ }^{12} \mathrm{~A}$ transient disturbance in liver function tests at presentation is characteristic of Legionnaires' disease, but the development of hepatic necrosis is not a feature described before. ${ }^{1-7}$ However, hepatic necrosis has been described in mice innoculated intraperitoneally with legionella. ${ }^{13}$

Finally, in this case peritoneal aspiration failed to diagnose a fatal bleed from splenic rupture. Peritoneal lavage has proved its worth in patients with possible abdominal trauma, but a simple peritoneal tap when negative is valueless. ${ }^{14}$ The patient's condition, however, deteriorated so rapidly that he died before an emergency laparotomy could proceed.

\section{Acknowledgement}

We thank Dr I.E. West, Consultant Forensic Pathologist and Head of Department of Forensic Medicine, United Medical School of Guy's and St Thomas's Guy's Hospital, London SE1 9RT for carrying out the post mortem.

\section{References}

1. Edelstein, P.H. \& Meyer, R.D. Legionnaire's disease - A Review. Chest 1985, 1: 114-119.

2. Keys, T.F. Legionnaire's disease - A review of the epidemiology and clinical manifestations of a new recognized infection. Mayo Clin Proc 1980, 55: 129-137.

3. Hernandez, F.J., Kirby, P.D., Stanley, T.M. \& Edelstein, P.H. Legionnaire's disease - postmortem pathological findings of 20 cases. Am J Clin Pathol 1980, 73: 488-495.

4. Balows, A. \& Fraser, D.W. (eds) International symposium on Legionnaires' disease. Ann Intern Med 1979, 90: 489-703.

5. Theodore, T.F., Tsai, M.D., David, R. et al. Legionnaires' disease: clinical features of the epidemic in Philadelphia. Ann Intern Med 1979, 90: 509-577.

6. Winn, W.C., Glavin, F.L., Perl, D.P. et al. The pathology of Legionnaires' disease. Fourteen fatal cases from the 1977 outbreak in Vermont. Arch Pathol Lab Med 1978, 1102: $344-350$.

7. Kirby, B.D., Synder, K.M. \& Meyer, R.D. Legionnaires' disease: clinical features of 24 cases. Ann Intern Med 1978, 89: 297-309.

8. Jandl, J.H. Blood, Textbook of Haematology. Little, Brown \& Co., Boston, USA, 1987, pp. $421-423$.

9. Kissani, J.M. In: Anderson, W.A.D. (ed.) Pathology, 7th Edition. C.V. Mosby Co., St Louis, USA, 1977, pp. 1497-1499.

10. Robbins, S.L., Cottran, R.S. \& Kumar, V. Pathological Basis of Disease, third edition. W.B. Saunders, Philadelphia, 1984

11. Zafram, E.S., Ishak, E.G. \& Rudzki, C. Cholestatic and hepatocellular injury associated with erythromycin esters?: report of nine cases. Dig Dis Sci 1979, 24: 385-396.

12. Miller, D.J., Leeton, G.R., Webber, B.C. \& Saunders, S.S Jaundice in severe bacterial infection. Gastroenterology 1976 , 17: 94-97.

13. Hedlond, E.W., McGann, V.G., Copeland, D.S. et al. Immunological protection against Legionnaires' disease bacteria in the AKR/J mouse. Ann Intern Med 1979, 90: 676-679.

14. Dudley, H.A.F. Hamilton Bailey's Emergency Surgery, 11 th Edition. Wright, Bristol, UK, 1986, chapter 40, pp. 391-410. 